REGARDS

SUR L'ECONOMIE ALLEMANDE

BULLETIN ECONOMIQUE DU CRAC
Regards sur l'économie allemande

Bulletin économique du CIRAC

$81 \mid 2007$

Varia

\title{
Hambourg, métropole portuaire internationale
}

\section{Michèle Weinachter}

\section{OpenEdition}

\section{Journals}

Édition électronique

URL : http://journals.openedition.org/rea/654

DOI : $10.4000 /$ rea. 654

ISBN : 978-2-8218-0858-4

ISSN : 1965-0787

Éditeur

CIRAC

Édition imprimée

Date de publication : 1 mai 2007

Pagination : 15-22

ISSN : 1156-8992

Référence électronique

Michèle Weinachter, " Hambourg, métropole portuaire internationale », Regards sur l'économie

allemande [En ligne], 81 | mai 2007, document 2, mis en ligne le 01 mai 2009, consulté le 19 avril 2019. URL : http://journals.openedition.org/rea/654; DOI : 10.4000/rea.654 


\section{Hambourg, métropole portuaire internationale}

\section{Michèle Weinachter}

Hambourg respire. Après l'annonce, fin février, du plan «Power 8 » chez Airbus, l'avenir de la ville comme site de production de l'avionneur semble assuré. II faut dire que la firme et l'aéronautique sont, depuis un certain temps déjà, étroitement associées à l'identité de la ville, au même titre que son port, ses docks et ses containers. La ville-Etat de Hambourg se veut être «Das Tor zur Welt ", la porte qui ouvre sur le monde. Ce port maritime, de loin le plus grand d'Allemagne, lui a donné, depuis des siècles et la glorieuse époque de la Hanse, son caractère cosmopolite. L'économie du Land, et notamment le secteur de la logistique, profitent aujourd'hui pleinement de la mondialisation et des importantes mutations géopolitiques survenues en Europe depuis quinze ans. Hambourg est également un grand site des médias et des nouvelles technologies associées. Pour conforter sa position parmi les grandes métropoles européennes, elle œuvre à la réalisation d'une stratégie de développement placée sous le mot d'ordre offensif « Metropole Hamburg - Wachsende Stadt » : Hambourg, une métropole en essor. Encore devra-t-elle se donner les moyens de cette politique.

Hambourg est située au Nord de l'Allemagne, entre les Länder de Basse-Saxe et du Schleswig-Holstein, à la confluence de l'Alster, du Bille et de l'Elbe. Comme Berlin et Brême, elle est la fois une ville et un Land, une ville-Etat (Stadtstaat). Hambourg compte 1,7 million d'habitants, ce qui en fait la $2^{\mathrm{e}}$ ville allemande après Berlin, pour une superficie de $755 \mathrm{~km}^{2}$, soit 2,5 fois celle de Londres, mais avec une densité de population inférieure de moitié (2 310 hab./ $\mathrm{km}^{2}$ ). Depuis 1991, Hambourg forme, en association avec 12 arrondissements des deux Länder voisins et deux arrondissements partenaires du Mecklembourg, qui regroupent au total près de 800 communes, la Région métropole Hambourg. C'est l'une des onze Régions métropole d'Allemagne - ces entités désormais considérées comme centrales dans le cadre de la stratégie de Lisbonne, les mises en réseau régionales devant permettre de mieux affronter la concurrence internationale. Cet ensemble structuré le long de l'Elbe compte près de 4,3 millions d'habitants. II s'étend de Cuxhaven au nord-ouest, à l'extrémité de l'estuaire du fleuve, jusqu'aux frontières du Land de Saxe-Anhalt au sud-est, en passant par l'Elbland et la lande de Lunebourg.

Hambourg est une ville-Etat, ...

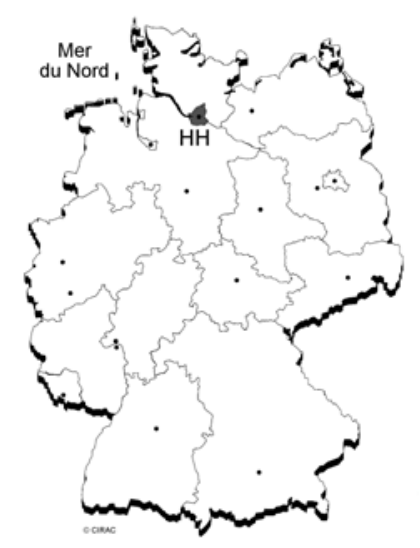

La ville de Hambourg a été fondée au IX ${ }^{e}$ siècle sous Charlemagne. Le commerce prend son essor au XIII ${ }^{e}$ siècle. En 1321, Hambourg devient membre de la Ligue Hanséatique, ce qui a fortement structuré son identité, comme l'indique aujourd'hui encore son nom officiel : Freie und Hansestadt Hamburg (ville libre et hanséatique de Hambourg; abréviation : HH). En 1515, elle obtient le statut de «ville libre d'Empire ». Elle est alors l'une des cités les plus riches d'Europe. Depuis 1949, Hambourg est un Land autonome, dirigé depuis 7 ans par un maire CDU, Ole von Beust, qui dirige également le Gouvernement du Land, nommé "Senat ».

L'architecture de la ville se caractérise par un mélange de styles et de matières, le plus typique étant le style de construction du début du $X X^{e}$ en brique rouge, caractéristique du Nord de l'Allemagne, que l'on retrouve dans la partie ancienne des docks. L'ensemble est très marqué par la présence de l'eau, de nombreux canaux traversent Hambourg, l'Alster formant jusqu'au cœur de la ville un lac artificiel. C'est surtout le développement de son célèbre port au bord de l'Elbe, à $110 \mathrm{~km}$ de la Mer du Nord, qui marque l'identité de la ville.

L'Elbe (1 $165 \mathrm{~km}$ de long) est, après le Rhin, le deuxième fleuve navigable ... située à l'embouchure de l'Elbe d'Allemagne par son importance. Elle prend sa source en République tchèque dans les monts des Géants et se jette dans la mer du Nord à Cuxhaven après 
Second port maritime d'Europe après Rotterdam

Une économie diversifiée

un long estuaire d'une centaine de kilomètres sur lequel se trouve Hambourg. Elle possède un vaste bassin fluvial et est au centre d'un ensemble de canaux constituant un important réseau de communications entre Rhin et Baltique. La Moldau qui passe par Prague se jette elle-même dans l'Elbe, et avec la réunification de l'Europe, la circulation de marchandises sur l'axe Prague-DresdeMagdebourg-Hambourg s'est considérablement développée, contribuant à l'extension des activités du port de Hambourg.

C'est grâce à ce dernier que la ville est devenue très tôt une métropole commerciale internationale. Après New-York, Hambourg est la ville qui détient le plus grand nombre de consulats : près d'une centaine. Elle est aussi le siège du Tribunal maritime international des Nations-Unies. Le port de Hambourg est le $2^{\mathrm{e}}$ port maritime d'Europe après Rotterdam. Chaque année, plus de 12000 navires y jettent l'ancre. Avec ses $87 \mathrm{~km}^{2}$, il occupe un dixième de la surface de la ville. Près de 140000 emplois en dépendent directement ou indirectement.

Si le port conserve un rôle central dans l'économie de la ville et de la région, d'autres activités se sont largement développées, notamment dans le domaine de la logistique, de la technologie, des médias, et des services. Une économie moderne, qui génère plus d'un million d'emplois, avec un PIB de 86 milliards $€$ en 2006 (3,73\% du PIB allemand). C'est plus que Berlin (80,3 milliards $€$ ) qui compte pourtant deux fois plus d'habitants. Parmi les 500 entreprises qui génèrent le plus fort $\mathrm{CA}$ en Allemagne, 39 ont leur siège à Hambourg. A côté des grands noms comme Airbus, Beiersdorf, Hapag-Lloyd, Helm, Olympus, Otto Versand, Panasonic, Tchibo ou de grands groupes de presse comme Gruner+ Jahr ou Bauer, Hambourg compte également de nombreuses PME performantes sur le marché international.

Création de richesse par secteurs en 2006 (en milliards $€$ )

\begin{tabular}{|c|c|c|c|c|c|}
\hline & Total & Agri-, sylviculture, pêche & Industrie (dont BTP) & $\begin{array}{c}\text { Commerce, hôtellerie, } \\
\text { transports }\end{array}$ & Autres services \\
\hline Hambourg & 77,63 & $0,14(0,2 \%)$ & $14,18(18,3 \%)$ & $21,26(27,4 \%)$ & $42,04(54,1 \%)$ \\
\hline Allemagne & 2079,00 & $20,09(1,0 \%)$ & $622,03(29,9 \%)$ & $380,41(18,3 \%)$ & $1056,47(50,8 \%)$ \\
\hline
\end{tabular}

Source des données : Statistische Ämter des Bundes und der Länder; pourcentages calculés par nos soins et arrondis.

Une industrie de services

Même si aujourd'hui, moins d'un actif sur six travaille dans le secteur secondaire, l'industrie reste, ici comme ailleurs, un moteur de développement pour les autres secteurs, en particulier celui des services, et pour l'innovation technologique. Hamburg demeure, avec Munich et Stuttgart, l'une des grandes métropoles industrielles de l'Allemagne. Le nombre d'emplois dans ce secteur a même augmenté ces dernières années pour certaines activités, comme l'aéronautique. Les biens d'investissement représentent $33 \%$ de la production industrielle, viennent ensuite le bâtiment $(22 \%)$, les biens de production, l'aéronautique (10\%), l'agro-alimentaire (8\%) et les biens de consommation (7\%).

Répartition des actifs par secteur en 2005 (en milliers)

\begin{tabular}{|lrrrrr|}
\hline & Total & Agri-, sylviculture, pêche & Industrie (dont BTP) & $\begin{array}{c}\text { Commerce, hôtellerie, } \\
\text { transports }\end{array}$ & \multicolumn{1}{c|}{ Autres services } \\
Hambourg & 802 & $6(0,8 \%)$ & $145(18,1 \%)$ & $238(29,7 \%)$ & $413(51,5 \%)$ \\
Allemagne & 36566 & $867(2,4 \%)$ & $10869(29,7 \%)$ & $8504(23,3 \%)$ \\
\hline
\end{tabular}

Source des données : Statistische Ämter des Bundes und der Länder; pourcentages calculés par nos soins et arrondis.

Une industrie vouée à l'origine aux activités portuaires
Historiquement, l'industrie de Hambourg s'est tout d'abord développée en lien avec les activités portuaires: importation et transformation de matières premières, pour le marché intérieur ou pour l'export. Les entreprises industrielles liées aux activités maritimes, comme la construction de bateaux, de machines ou de moteurs, l'électrotechnique et le bâtiment constituent, aujourd'hui encore, le deuxième pilier de l'industrie hambourgeoise. Un troisième pilier s'est développé, celui des activités non liées au port, comme celui des biens de consommation, grâce à la demande d'une population croissante dans la région. Plus ré- 
cemment enfin se développe un réseau de PME dans le secteur des éco-industries, en forte croissance.

Plus de trois quarts des actifs travaillent dans le secteur des services. Le commerce et la logistique y occupent une place centrale, avec 40000 entreprises et 200000 emplois. Ils font eux-mêmes appel à des services associés à leurs activités, comme les banques et assurances. C'est à Hambourg que la première bourse allemande avait ouvert ses portes en 1558, la première banque privée en 1590 , et la première compagnie d'assurance en 1676. Aujourd'hui, la ville occupe la deuxième place derrière Francfort pour le nombre de banques (160), avec 30000 employés. Pour les assurances, Hambourg est numéro 3 en Allemagne (20000 emplois), certains assureurs étant spécialisés dans le transport de marchandises, comme la Junge \& Co. Versicherungsmakler $\mathrm{GmbH}$.

Le développement économique de la ville a été depuis deux décennies largement dynamisé par l'évolution de la situation internationale. Depuis des siècles, Hambourg constitue pour les marchandises en provenance de tous les continents la porte d'entrée vers les marchés européens. Depuis 1986, la ville est jumelée avec Shanghai, le plus grand port de commerce chinois. L'accélération de la globalisation a donné à Hambourg une forte dynamique de croissance. L'évolution de la situation géopolitique européenne à la fin du $X X^{e}$ siècle a joué dans le même sens. Avec la nouvelle vague d'élargissement de l'UE en 1995 (Autriche, mais aussi Finlande et Suède), l'ensemble des pays scandinaves étaient désormais dans I'Union. En 2004 et 2007, outre Malte et Chypre, onze nouveaux Etats d'Europe centrale et orientale rejoignaient le grand marché unique. Hambourg a pu ainsi développer ou renouer des liens parfois très anciens avec les pays d'Europe centrale d'une part, avec toute la zone de la Mer Baltique 'réunifiée', d'autre part - une zone qui connaît une forte croissance économique et constitue un marché attractif de plus de 75 millions d'habitants.

Le commerce extérieur se développe en conséquence. En 2005, les importations ont augmenté de 10,5\%, et les exportations de $8,3 \%$. Les pays d'Asie sont devenus l'un des partenaires les plus importants de Hambourg. Le commerce avec la Chine notamment se développe de façon exponentielle (la croissance des exportations vers la Chine a été de $67,2 \%$ en 2005, atteignant un total de 1,55 milliard $€$ ). Mais en volume, l'échange de marchandises à l'intérieur de l'Union européenne occupe, de loin, la première place, et il va continuer de croître avec le développement des nouveaux Etats membres. Enfin, le continent américain est également un partenaire important.
Services :

commerce, banque, assurance

Des activités dynamisées par les élargissements européens et la mondialisation

Croissance exponentielle du commerce avec la Chine

Principaux partenaires commerciaux de Hambourg en 2005 (en milliards $€$ )

\begin{tabular}{|lcccc|}
\hline & UE & Continent américain & Continent asiatique & Total \\
Exportations & $15,41(67,8 \%)$ & $2,83(12,5 \%)$ & $2,77(12,2 \%)$ & 22,73 \\
Importations & $22,16(48,4 \%)$ & $7,51(16,4 \%)$ & $10,43(22,8 \%)$ & 45,77 \\
\hline
\end{tabular}

Source des données : Statistische Ämter des Bundes und der Länder; pourcentages calculés par nos soins et arrondis.

\section{Trois piliers de développement économique}

Cette évolution de la situation internationale a permis à Hambourg de conforter sa place en tant que pôle leader dans le domaine de la logistique pour l'ensemble de l'Europe du Nord. La logistique est en effet, à côté de l'aéronautique et de la branche MITT (médias, technologies de l'information, télécommunications), l'un des trois piliers du développement économique de la métropole hanséatique.

Dans le domaine de la logistique, le port de Hambourg se classe numéro deux en Europe, et $8^{e}$ au plan mondial. En 2006, avec une croissance de $9,6 \%$, de nouveaux records ont été battus : plus de 135 millions de tonnes de marchandises, soit 8,9 millions de containers (TEU) ont transité par le port. Sur ce total,
Hamburg, la plate-forme logistique du Nord de l'Europe... 
... grâce à la qualité des infrastructures et à l'intermodalité

La logistique, secteur en pleine expansion

L'aéronautique, $2^{\mathrm{e}}$ pilier du développement économique plus de la moitié (4,8 millions de containers) concerne le commerce avec l'Asie. De fait, la Chine est désormais le principal partenaire commercial de Hambourg, où sont installées plus de firmes chinoises (désormais plus de 400) que dans n'importe quelle autre ville d'Europe continentale. Le site internet du port (www.hamburg-hafen.de) est accessible en allemand, anglais, polonais, russe, tchèque et chinois, ce qui est significatif de l'évolution en cours. Plus d'un tiers des marchandises débarquées sur le port a pour destination finale l'une des communes de la Région métropole, où 230000 actifs travaillent dans le secteur de la logistique, dont 154000 à Hambourg même. Ils génèrent $11 \%$ de la création de richesse de la ville-Etat.

Les marchandises peuvent, après leur arrivée à Hambourg, être acheminées vers leur destination par voie fluviale, ferroviaire, routière et aérienne. Ces différents modes de transport se livrent d'ailleurs une rude concurrence. Le fret ferroviaire détient actuellement $70 \%$ du marché, le port de Hambourg étant l'un des principaux clients de la Deutsche Bahn. II faut dire que Hambourg est le principal point d'interconnexion ferroviaire du Nord de l'Allemagne: cinq grandes lignes et deux lignes régionales s'y croisent, assurant des liaisons fréquentes avec Berlin, Hanovre, Brême/la Ruhr, Flensburg/le Jütland, Lübeck/Copenhague et, au-delà, avec les principaux centres économiques européens. La région bénéficie également d'un excellent maillage de canaux reliant entre eux les fleuves, et les mers. Le Elbe-Seiten-Kanal $(115 \mathrm{~km})$ notamment permet de relier Hambourg à l'ensemble du réseau fluvial allemand. En l'empruntant, les bateaux de fort tonnage ont accès au Mittellandkanal $(326 \mathrm{~km})$, le plus grand canal transversal d'Allemagne. Le Nord-Ostsee-Kanal $(99 \mathrm{~km})$ relie pour sa part la Mer du Nord à la Baltique. Le transport routier devrait quant à lui se développer à la faveur de l'amélioration prévue du réseau routier et autoroutier de Hambourg, considéré comme vieilli et insuffisamment développé. Hambourg compte néanmoins plus de 1700 entreprises de transport routier. Le fret aérien dispose pour sa part d'infrastructures et d'une logistique ultra-modernes (système ELWIS) à l'aéroport, situé à $8 \mathrm{~km}$ du centre-ville.

Pour conforter la place de Hambourg dans ce secteur (qui devrait, d'ici 2015, créer environ 15000 emplois supplémentaires) a été créée en 2006 la LogistikInitiative Hamburg. Ce réseau qui regroupe les acteurs de la branche et les pouvoirs publics cherche à en accroître la compétitivité, en favorisant notamment la synergie avec les instituts de recherche et de formation. Hambourg propose ainsi de nombreuses formations dans le domaine de la logistique, y compris un MBA à la Hamburg School of Logistics, afin de fournir aux entreprises la main d'œuvre dont elles ont besoin.

En Allemagne du nord, un cluster regroupant plus de 1900 entreprises et institutions s'est formé dans le domaine de l'aéronautique civile. II a pour centre Hambourg qui est, après Seattle et Toulouse, le troisième plus grand site de production dans le monde. 32000 personnes travaillent dans cette branche. Les trois plus gros employeurs sont Airbus Deutschland $\mathrm{GmbH}$, la Lufthansa Technik AG, filiale de la Lufthansa, et l'aéroport de Hambourg. Ils travaillent en collaboration avec leurs fournisseurs, plus de 300 PME du secteur qui emploient près de 9000 personnes dans la région. Au-delà de la construction, de la maintenance et de l'exploitation des avions, cette branche génère de nombreux emplois dans les services associés : ingénierie, conseil, design, ou encore dans l'électrotechnique, l'électronique, l'optique, la construction mécanique et le bâtiment. Les grandes entreprises du secteur ont établi des liens étroits avec certaines institutions de formation supérieure et de recherche, comme par exemple Airbus avec I'Université Technique de Hamburg-Harburg (TUHH), ou encore la Lufthansa Technik Logistik avec I'Institut Fraunhofer IIS (Institut für Integrierte Schaltungen, Institut Fraunhofer pour les circuits intégrés).

Ici encore, il s'agit de maintenir la compétitivité et de favoriser recherche et innovation. Hambourg est par exemple l'un des principaux pôles allemands de re- 
cherche sur la filière hydrogène et ses applications dans l'aviation et la marine (voir REA 66/04). La ville soutient activement ces coopérations dans le cadre de I'Initiative Luftfahrtstandort Hamburg (Hambourg, site de production pour l'aéronautique), lancée en 2001.

La société Flughafen Hamburg $\mathrm{GmbH}$ est exploitant de l'aéroport de la ville. Au total, celui-ci emploie plus de 12000 personnes. La capacité de l'aéroport, situé en zone urbaine, reste limitée, ce qui est considéré comme un handicap pour l'économie de la ville. II a cependant battu des records en 2006, où il a enregistré à la fois sa plus forte croissance et un nombre de passagers encore jamais atteint auparavant : avec 11,95 millions de passagers, il est désormais le $4^{\mathrm{e}}$ aéroport d'Allemagne, et dessert près de 120 destinations. Un programme de modernisation et d'extension est en cours de réalisation. Ce projet, "HAM 21 ", qui prévoit plus de 350 millions $€$ d'investissements, est le plus important depuis la création de l'aéroport. II prévoit la construction d'un nouveau terminal, d'un parking de 2200 places, d'une nouvelle galerie commerciale, et l'amélioration des voies d'accès à l'aéroport, avec notamment un raccordement au réseau de trains rapides de la ville. Le nouveau tronçon permettra de rejoindre la gare du centre-ville en 23 minutes. L'aéroport devra ainsi pouvoir accueillir jusqu'à 15 millions de passagers par an.

L'aéroport héberge également la Lufthansa Technik AG, filiale de la Lufthansa AG. Elle emploie plus de 7000 personnes à Hambourg. Avec un CA de plus de 3 milliards $€$ par an, elle est la plus grosse entreprise de maintenance, réparation et révision d'avions au monde, au service de 340 compagnies aériennes.

Mais l'élément le plus dynamique de ce cluster, et son plus gros employeur, est Airbus. Hambourg est en effet le plus gros site de production de l'avionneur en Allemagne. Dès 1985, Hambourg comptait $20 \%$ des employés de la compagnie. Sur les 52000 salariés du groupe, 12500 travaillent désormais à l'usine de Hamburg-Finkenwerder. C'est à Hambourg que se fait depuis 1993 l'assemblage final des modèles A318, A319, A321. Hambourg est également associé à la construction du nouvel avion géant $\mathrm{A} 380$, avec notamment la production de certaines parties du fuselage et l'équipement des cabines. Depuis plusieurs années, les pouvoirs publics ont investi près d'un milliard d'euros dans un vaste programme de développement des infrastructures liées à la production et à la livraison de l'A380. Le prolongement de la piste de décollage, contre lequel les écologistes avaient livré un long combat acharné, sera finalement achevé courant 2007. Depuis 2000, près de 4000 emplois ont été créés.
3 grands acteurs pour l'Initiative : la Flughafen Hamburg $\mathrm{GmbH}, \ldots$

... la Lufthansa Technik AG...

... et Airbus

\section{POWER 8 préserve le site de production de Hambourg}

Dans ce contexte, l'annonce depuis l'automne 2006 d'un vaste plan de restructuration chez l'avionneur, dû notamment au retard pris dans la production de l'A380, ne pouvait que susciter de vives inquiétudes. 10000 suppressions d'emplois étaient envisagées, la vente de certains sites, la « recherche de partenariats » pour d'autres. Finalement, le plan Power 8, dévoilé le 28 février dernier, a été accueilli avec un certain soulagement à Hambourg. Certes, ici aussi, de nombreux emplois seront supprimés (plus de 1000 sur les 3700 suppressions de postes prévues en Allemagne).

Mais, contrairement à d'autres sites de Basse-Saxe ou du Bade-Wurtemberg, Hambourg n'est pas remis en cause comme site de production. Les autorités de la ville estiment qu'à moyen et long terme, il se trouve même renforcé. Hambourg continuera notamment, ce qui est perçu comme décisif, à participer au développement des produits Airbus, et tous les engagements pris antérieurement par EADS seront tenus. L'usine de Finkenwerder reste chargée du fuselage et de certains équipements de l'A380, ainsi que de l'assemblage des A318, A319 et A321. De plus, Hambourg obtient immédiatement une troisième ligne de montage pour le modèle A320 (qui était jusque-là exclusivement assemblé à Toulouse). La nouvelle version de l'A320 sera également confiée à Hambourg. L'obtention de la troisième ligne de montage pour l'A320 a été parfois présentée comme une concession faite à Hambourg "en échange » de la concentration de l'assemblage de l'A350 sur le site de Toulouse. En réalité, cette décision avait été prise dès 2000 , mais la mise en œuvre avait jusqu'alors toujours été différée. Hambourg a pu sortir ce 'joker' lors des négociations récentes autour de Power 8.

Le scénario-catastrophe redouté par certains a donc été évité, et Hambourg restera, à côté de Toulouse, le principal site de production d'Airbus. Le Senat de la ville-Etat veut y voir aussi le résultat de son action de soutien en faveur du groupe : outre les investissements évoqués ci-dessus, Hambourg s'est engagé financièrement dans le groupe EADS lors du récent retrait de DaimlerChrysler, qui a cédé $7,5 \%$ du capital. Hambourg est désormais le Land le plus engagé financièrement dans EADS. Mais Airbus n'a pas encore surmonté la crise actuelle, et les inquiétudes quant à l'avenir du groupe européen demeurent. 
$3^{\mathrm{e}}$ pilier de l'économie : médias et télécommunications
La branche des médias, des technologies de l'information et des télécommunications constitue le $3^{\mathrm{e}}$ pôle de développement de l'économie hambourgeoise. Hambourg est la capitale allemande de la presse depuis près de quatre siècles. Son premier hebdomadaire a paru en 1618. Aujourd'hui, deux des quatre plus grandes maisons de presse ont leur siège à Hambourg : Gruner + Jahr (filiale de Bertelsmann, Gütersloh) et le groupe Bauer. S'y ajoute le siège central de la rédaction du quotidien allemand au plus fort tirage, Bild, publié par les éditions Axel Springer (Berlin). Ensemble, ces trois groupes détiennent près de $50 \%$ du marché de la presse grand public (voir REA 75/06). C'est ici aussi qu'est produit I'hebdomadaire Der Spiegel. C'est également à Hambourg qu'est installée la plus grande agence de presse allemande, la dpa (Deutsche Presse Agentur). Mais le monde des médias ne se résume pas aux journaux, à la télévision et Internet, même si ces trois branches comptent près de 6000 entreprises. II implique de plus en plus d'acteurs, par exemple dans la publicité (secteur où Hambourg fait figure de 'capitale' allemande), le design ou le marketing. Hambourg incarne la complexité de ce secteur en pleine croissance : la ville compte plus de 11000 entreprises dans le monde des médias, soit $75 \%$ de plus qu'en 1995. Le secteur (plus de 70000 actifs) est le $4^{\mathrm{e}}$ plus gros employeur de la ville et génère un $C A$ de plus de 25 milliards $€\left(3^{\mathrm{e}}\right.$ position dans l'économie de la ville). Mais on arrive à près de 17000 entreprises et 120000 actifs si on ajoute le domaine des télécommunications et des technologies de l'information, qui fournit matériel et services pour les nouveaux médias numériques et les nouvelles techniques de communication. Ce secteur, au CA de 9 milliards $€$ en 2005 , devrait continuer à connaître une forte croissance. Le Senat soutient activement cette branche, et s'est fixé pour objectif de développer à Hambourg un "Kompetenz-Cluster IT » (pôle de compétitivité TIC), dans le cadre de la stratégie globale de développement de la ville présentée en 2002.

\section{Une stratégie de développement clairement affichée}

Cette stratégie à moyen et long terme, baptisée «Metropole Hamburg - Wachsende Stadt ": Hambourg, une métropole en essor, vise à conforter la position de la région parmi les grandes métropoles internationales. Elle a défini quatre objectifs principaux, quantitatifs mais aussi qualitatifs : une croissance durablement supérieure à celle des villes comparables, l'augmentation du nombre d'habitants, une plus forte attractivité et le renforcement du rôle de métropole, la préservation de la qualité de vie dans le cadre d'un développement durable. Du point de vue économique, cela se traduit par un certain nombre de priorités : un soutien actif aux 120000 PME de la Région métropole ; une politique de cluster dans les secteurs innovants et/ou à forte croissance ; une amélioration de la coopération entre les différentes instances de la Région métropole en vue de soutenir la croissance ; enfin, la réalisation du grand projet d'urbanisme « $\mathrm{Ha}$ fencity 》(cité portuaire).

\section{La Hafencity, emblème de l'expansion de la ville}

Ce projet futuriste, actuellement le plus grand chantier urbain en Europe, doit changer le visage du centre-ville, qui va connaître une extension de surface de $40 \%$ (voir www.hafencity.com). L'achèvement des travaux (5 milliards $€$ d'investissements) est prévu pour 2020. La Hafencity mêlera sur 155 hectares logements, bureaux, centre de foires et de congrès, activités économiques dans tous les secteurs (40 000 emplois). Hambourg entend ainsi attirer de nombreuses entreprises ; SAP, Jungheinrich ou Unilever ont déjà annoncé leur installation.

La Hafencity se veut l'incarnation du dynamisme de Hambourg et de son économie. Son rayonnement passera également par une offre culturelle et de loisirs remarquable : outre plusieurs musées et un aquarium, la Hafencity accueillera l'Elbphilarmonie, censée devenir l'une des dix meilleures salles de concert du monde. Son coût, objet de polémiques, est finalement estimé à 240 millions $€$, soit 50 millions de plus que prévu. Le bâtiment sera en partie financé par des dons des citoyens, d'entreprises et de fondations (65 millions $€$ ont déjà été récoltés). Les travaux de construction ont commencé début avril 2007. Son achèvement est prévu pour mai 2010.

L'Elbphilarmonie, à l'architecture résolument contemporaine, doit devenir le nouveau symbole de la ville-Etat. 
Ces projets de développement devraient également profiter à la branche du tourisme, qui génère d'ores et déjà près de 100000 emplois directs et indirects, avec une capacité hôtelière de 30000 lits. Avec 6,4 millions de nuitées et 55 millions de visiteurs au total en 2005, Hambourg se classe en treizième position parmi les grandes villes européennes - devant Lisbonne, Bruxelles, Stockholm ou Copenhague. D'ici 2010, Hambourg aspire à se classer parmi les dix premières destinations européennes. Pour l'instant, le point faible reste le nombre insuffisant de touristes étrangers ; ils représentent seulement $20 \%$ du total. La ville mise ici sur la multiplication des liaisons aériennes intercontinentales directes et une meilleure desserte de Hambourg par les compagnies à bas coûts. La ville veut également conforter sa position de premier port d'accueil pour les croisières touristiques - un marché en pleine expansion. En 2006, le terminal pour bateaux de croisière a accueilli plus de 60 navires, dont le Queen Mary 2 et le MS Europa. Dans le cadre du projet Hafencity, la capacité d'accueil du Hamburg Cruise Center doit encore être améliorée.

\section{Des performances économiques contrastées}

La situation économique de la ville-Etat s'est nettement améliorée en 2006. Le Senat veut voir dans ces bons résultats économiques les premiers succès de sa politique de développement. En 2006, la croissance du PIB du Land a été remarquable. Avec $+2,9 \%$, Hambourg se classe en $3^{\mathrm{e}}$ position après la Saxe $(+4 \%)$ et le Bade-Wurtemberg $(+3,5 \%)$, la moyenne allemande se situant à $+2,5 \%$ (ces données qui datent d'avant la révision effectuée fin février 2007 par Destatis, et qui porte la croissance de la RFA à 2,9\%, n'ont pas encore été actualisées en ce qui concerne les Länder). Le chômage a connu une baisse de $13,6 \%$ entre février 2006 et février 2007, et le taux s'élève désormais à $10 \%$ de la population active. Hambourg a certes profité d'un contexte général favorable, marqué par le redémarrage de l'économie outre-Rhin. Parmi les évolutions encourageantes, on relève une augmentation du nombre d'actifs de 1,2\% entre 2003 et 2005. Hambourg arrive ici en $1^{\text {ère }}$ position, à en croire l'étude comparative INSM/Wirtschaftswoche Dynamik-Ranking de 2006, qui classe les Länder allemands en fonction de l'évolution d'un certain nombre d'indicateurs économiques et structurels entre 2003 et 2005 . Hambourg continue également à enregistrer de bons résultats en ce qui concerne le PIB par habitant (avec $45992 €$ par tête, il est largement au-dessus de la moyenne des 3 villes-Etats : $35463 €$ ) et la productivité (76 $084 €$ par actif ; moyenne des 3 villes-Etats : $64017 €)$.

Mais l'économie hambourgeoise n'enregistre pas que de bons résultats. Si le taux de chômage est certes désormais dans la moyenne allemande, il est bien supérieur par exemple au taux de chômage de la Bavière ou du Bade-Wurtemberg (autour de $6 \%$ ), ou même à la moyenne ouest-allemande du début $2007(8,4 \%)$. Et au total, Hambourg n'arrive qu'en $10^{\mathrm{e}}$ position parmi l'ensemble des Länder dans le classement 2006 établi par l'étude INSM/Wirtschaftswoche Dynamik-Ranking évoquée ci-dessus. La part des dépenses publiques consacrée aux investissements a notamment chuté de 3,1\% entre 2003 et 2005. En 2005, elle n'atteignait que 10,8\% du budget - la ville arrive ici en avant-dernière position pour toute l'Allemagne.

II faut dire que la ville-Etat est lourdement handicapée par le poids de sa dette : Hambourg fait partie du trio de tête des Länder les plus endettés d'Allemagne si on regarde le taux d'endettement par habitant (voir REA 79/06). Le problème est tel que le Senat explore des pistes assez radicales pour sortir de la crise. Depuis 2005, le maire et ministre-président Ole von Beust envisageait même de vendre la partie du Port et de ses installations appartenant à la ville, la Hamburger Hafen und Logistik AG (HHLA). Le projet avait suscité chez certains une vive indignation, tant le port est perçu comme faisant partie du patrimoine collectif. Après l'échec des négociations avec la Deutsche Bahn en 2006, le
Un tourisme en expansion

Une croissance de $2,9 \%$ en 2006

Une nette faiblesse de l'investissement public

Hambourg est l'un des Länder les plus endettés 
Senat était à la recherche d'une autre solution. Finalement, mi-mars 2007, l'introduction en bourse de $30 \%$ des parts de la société a été annoncée. La nouvelle a été accueillie avec soulagement - notamment par les syndicats qui menaçaient de déclencher une grève et de paralyser le port. Mais le problème de la dette demeure, et tant qu'il n'est pas réglé, la marge de manœuvre de la ville pour la mise en œuvre de sa stratégie de développement s'en trouve considérablement restreinte. Cette question sera sans nul doute au centre des débats qui précéderont les échéances électorales régionales de 2008.

ConCERnANT L'AMBition DE HAMBouRg de conforter sa place parmi les grandes métropoles européennes, les années qui viennent vont être décisives. Les axes de développement ont été clairement fixés. La dynamisation des infrastructures doit conforter les atouts territoriaux, des pôles de compétitivité émergent dans les secteurs de la technologie et de la communication, le tout contribuant au rayonnement urbain et culturel de la métropole hanséatique. Par son positionnement international, la ville-Etat profite pleinement des opportunités offertes par les élargissements récents de l'UE. Elle profite également de la globalisation et de l'essor de certaines branches qui en dépendent directement, y compris dans le domaine industriel, avec par exemple l'aéronautique. Au moment où Airbus connaît des difficultés et doit se restructurer, une embellie a semblé apparaître au plan commercial. En mars, l'avionneur européen a notamment reçu la promesse de deux commandes pour son futur long courrier A350 XWB, pour un montant record de 17,3 milliards $€$. Mais au même moment, une nouvelle menace se profile, qui pourrait bien, à relativement brève échéance, concerner Hambourg : la Chine vient de donner son feu vert au programme d'un avion gros-porteur civil susceptible de transporter plus de 150 passagers. Dans ce domaine comme dans d'autres secteurs industriels stratégiques, les Chinois vont, après avoir largement bénéficié de transferts technologiques des pays occidentaux, leur livrer une rude concurrence. Airbus, qui se partage pour l'instant avec Boeing un marché chinois en pleine croissance, a du souci à se faire. En revanche le port de Hambourg devrait pouvoir jouer pleinement le rôle de plaque tournante du commerce euro-chinois : la Chine vient de dépasser les Etats-Unis comme premier fournisseur de produits à l'UE.

\section{Indications bibliographiques}

Bertelsmann-Stiftung, Die Bundesländer im Standortwettbewerb 2005, Gütersloh, 2006 Bundesländer-Ranking, étude réalisée par IW Consult en partenariat avec l'Initiative Neue Soziale Marktwirtschaft et l'hebdomadaire Wirtschaftswoche, oct. 2006 (www.insm.de)

KROPP P., « Hamburg - Wirtschaft und Arbeitsmarkt im Metropolenvergleich », in Sozialer Fortschritt, vol. 11/12, 2006

Monitor Wachsende Stadt - Bericht 2006, édité par le Statistisches Amt für Hamburg und Schleswig-Holstein, Hamburg/Kiel, 2006, en coopération avec le Senat de la ville (accessible sur le site www.wachsende-stadt.hamburg.de)

www.hafen-hamburg.de

www.hamburg-economy.de

www.hamburg-logistik.com

www.luftfahrtstandort-hamburg.de

www.medien.hamburg.de

www.statistik-nord.de

www.statistikportal.de. 